\title{
Quality of life in patients with Marfan syndrome: a cross-sectional study of 102 adult patients
}

\author{
Caroline Andonian ${ }^{1,2,3}$, Sebastian Freilinger ${ }^{1}$, Stefan Achenbach ${ }^{4}$, Peter Ewert ${ }^{1}$, Ulrike Gundlach ${ }^{4}$, \\ Harald Kaemmerer ${ }^{1}$, Nicole Nagdyman ${ }^{1}$, Rhoia Clara Neidenbach ${ }^{1}$, Lars Pieper ${ }^{5}$, Jörg Schelling ${ }^{6}$, \\ Michael Weyand ${ }^{4}$, Jürgen Beckmann ${ }^{2,7,8}$
}

${ }^{1}$ Department of Congenital Heart Disease and Pediatric Cardiology, German Heart Center Munich, Technical University Munich, Munich, Germany; ${ }^{2}$ Department of Sport and Health Sciences, Chair of Sport Psychology, Technical University Munich, Munich, Germany; ${ }^{3}$ Sigmund Freud University, Vienna, Austria; ${ }^{4}$ Department of Cardiology, University of Erlangen, Erlangen, Germany; ${ }^{5}$ Department of Behavioral Epidemiology, Technical University of Dresden, Dresden, Germany; ${ }^{6}$ Faculty of Medicine, Ludwig Maximilians University (LMU) of Munich, Munich, Germany; ${ }^{7}$ School of Human Movement and Nutrition Sciences, University of Queensland, Australia; ${ }^{8}$ Health Research Institute, University of Limerick, Limerick, Ireland

Contributions: (I) Conception and design: C Andonian, S Freilinger, H Kaemmerer, L Pieper, RC Neidenbach, J Beckmann; (II) Administrative support: All authors; (III) Provision of study materials or patients: S Achenbach, P Ewert, U Gundlach, H Kaemmerer, N Nagdyman, J Schelling, M Weyand; (IV) Collection and assembly of data: C Andonian, S Freilinger, L Pieper, RC Neidenbach; (V) Data analysis and interpretation: C Andonian, S Freilinger, J Beckmann; (VI) Manuscript writing: All authors; (VII) Final approval of manuscript: All authors.

Correspondence to: Caroline Andonian, M.Sc. Department of Congenital Heart Disease and Pediatric Cardiology, German Heart Center Munich, Technical University Munich, Munich, Germany. Email: andonian@dhm.mhn.de.

Background: Marfan syndrome (MFS) is a genetically determined multiorgan disease that leads to severe physiological and psychological impairments in adult life. Little consensus exists regarding quality of life (QOL) in individuals with MFS. The present study sought to investigate QOL in a representative cohort of adults with MFS.

Methods: Patient-reported outcome measures from a representative sample of 102 adults with MFS $(39.3 \pm 13.1$ years of age; $40.2 \%$ female) were retrospectively analyzed and compared with those from adults with different congenital heart defects (CHD), at the German Heart Center Munich. QOL was assessed using the updated five-level version of the EQ-5D.

Results: Differences between both populations were analyzed. Subjects affected by MFS reported an overall reduced QOL. Compared to CHD patients, individuals with MFS scored significantly lower in the dimensions of pain/discomfort, anxiety/depression, mobility and usual activities $(\mathrm{P}<0.05)$.

Conclusions: Patients with MFS are at high risk for impaired QOL, especially in mental and physical domains. Psychosocial consequences of MFS cost resources for both, patients and professionals. Current findings highlight the great importance of additional psychological support to cope with disease-related challenges. Increased attention should be directed towards enhancing their subjective wellbeing to potentially improve their QOL and long-term health outcomes.

Keywords: Marfan syndrome (MFS); psychological situation; quality of life; prevention; EQ-5D

Submitted Aug 05, 2020. Accepted for publication Dec 18, 2020.

doi: $10.21037 / \mathrm{cdt}-20-692$

View this article at: http://dx.doi.org/10.21037/cdt-20-692 


\section{Introduction}

Marfan syndrome (MFS) is a genetically determined multiorgan disease that affects the connective tissue throughout the body, including the skeletal, ocular, pulmonary, cardiovascular, and central nervous systems. An estimated proportion of $0.002 \%$ to $0.017 \%$ of the population is affected by MFS (1). The most severe complications from a medical perspective include aneurysm formation and dissection of the aorta. Consequently, most patients are closely monitored, with serial cardiovascular assessments from early childhood (2-5). Additionally, patients with MFS often have to cope with skeletal or ocular abnormalities leading to a characteristic appearance, which is not only subjectively perceived by themselves, but is also visible to others $(6,7)$. Recent studies have indicated that the combination of physiological and psychological symptoms may lead to a decreased quality of life (QOL) in individuals with MFS (3).

Despite the growing interest in psychosocial consequences for patients with congenital heart defects (CHD), research on the particular psychological concerns of individuals with MFS is still scarce (8). While clinical research has traditionally focused on "hard" outcome measures, such as morality, morbidity, and functional status, the concept of QOL has become increasingly recognized as an important patient-reported outcome measure in the evaluation of care and treatment (9). Patients with MFS present an elevated risk for adverse psychosocial outcomes, including decreased QOL, particularly in the psychological domain (3,4,10-12). Some patients with MFS also experience symptoms of emotional distress, such as depression and anxiety $(4,7,13)$. Emotional distress can impact a patient's overall health and lead to increased cardiovascular morbidity and premature mortality $(14,15)$.

The present study aimed to (I) systematically assess QOL within a large sample of patients affected by MFS and (II) compare the results with findings on patients with other types of CHD. In an attempt to raise awareness for the psychosocial implications of MFS, this study should encourage clinicians to evaluate the psychological status of patients with MFS and, on the basis of this status, enhance treatment options. We present the following article in accordance with the STROBE reporting checklist (available at http://dx.doi.org/10.21037/cdt-20-692).

\section{Methods}

\section{Population}

The present study represents a subgroup analysis of the nation-wide VEMAH study (www.vemah.info) which constitutes the first large-scale attempt to comprehensively assess the health care situation of a large cohort of adults with congenital heart disease (ACHD) in Germany. The questionnaire-based survey was initiated and carried out by the Department of Congenital Heart Disease of the German Heart Centre Munich, Technical University Munich, and the Department of Cardiology, University of Erlangen. The insurance company "AOK Bayern" supported the study by sending out our questionnaires to their insured ACHD patients in Bavaria. The study was conducted in accordance with the Declaration of Helsinki (as revised in 2013) and under the approval of the Ethics Committee of the Technical University of Munich (157 / 16 S). Data collection took place between May 2017 and July 2020. Written informed consent was obtained from all participating patients before the start of documentation. Guidelines on good pharmacoepidemiologic practice and data protection guidelines were followed.

Patients were selected for sub-analysis according to the following inclusion criteria: (I) confirmed diagnosis of MFS; (II) participant age 18 years or older; (III) necessary physical, cognitive and language capabilities to complete self-report questionnaires; (IV) German speaking. Participants were excluded if they did not fulfill age requirements or had severely impaired cognitive abilities. Patients were consecutively included in the order that they presented at the institution and were not selected in prior.

\section{Measures}

Patient-reported outcome measures were assessed by a specifically devised questionnaire in cooperation with the Chair of Behavioral Epidemiology at the Technical University of Dresden and the German Heart Center Munich. The questionnaire was completed in person, online or by mail. Data collection took place between 2016 and 2019. QOL was measured using the updated fivelevel version of the EQ-5D (EQ-5D-5L) which provides a simple, generic measure of a patient's perceived health status. Research has shown that this version presents a significantly higher reliability and discriminatory power and reduces ceiling effects, , compared with the original EQ-5D (16). The EQ-5D-5L is a paper-based, selfcomplete questionnaire consisting of two sections: a descriptive system questionnaire and a visual analogue scale (VAS). The descriptive system compromises five dimensions: mobility, self-care, usual activities, pain/discomfort and 
anxiety/depression. The patient is asked to indicate his or her perceived impairments on a 5-point Likert scale ranging from "no problems" to "extreme problems/unable". Responses are coded as single-digit numbers expressing the severity of impairment on each dimension. Responses can be converted into a single weighted index score (EQ$5 \mathrm{D}$ index) using population preference scores. A value set for the EQ-5D-5L based on a representative sample of the German population has recently been developed (17). The EQ-VAS indicates a patient's overall health state on a vertical scale which ranges from 0 ("The worst health you can imagine") to 100 ("The best health you can imagine"). It therefore provides a quantitative measure of a patient's perceived health.

\section{Statistical analysis}

Statistical analysis was performed using SPSS 25.0 (IBM Inc., Armonk, NY, USA). Medical records were reviewed for patient demographics, cardiac and non-cardiac diagnoses. Statistical evaluations were pseudonymized and not linked to individual person. Descriptive measures were calculated for sociodemographic sample characteristics. Several logistic regression models were calculated to analyse the impact of MFS on descriptive QOL dimensions, using the EQ-dimensions of the EQ-5D-5L as dependent variables. Differences between the populations were evaluated using the chi-squared tests. $T$-tests were used for comparisons between mean values. Continuous data was expressed as mean \pm standard deviation, categorical or interval scaled variables as absolute numbers or percentages. The crosswalk-index-value (utility index) of the EQ5D$5 \mathrm{~L}$ was calculated using the German value set (17). All occurring $\mathrm{P}$ values and tests for significance were performed in a two-sided manner. Statistical significance was indicated by a $\mathrm{P}$ value $<0.05$.

\section{Results}

\section{Sample characteristics}

Out of 3,885 patients, a total of 102 patients with MFS were retained for the final analysis ( $40.2 \%$ female) (Table 1). The mean age of the patients with MFS was $39.3 \pm 13.1$ (range, 20-85) years. The remaining 3,783 ACHD [42.0 \pm 17.3 (range, 18-97) years; $46.6 \%$ female] were consulted to offer a point of reference consisting of non-MFS ACHD. Both populations were comparable in their age distribution, residential zone and insurance status.

\section{QOL in patients with MFS}

Table 2 shows the impact of MFS on all five QOL dimensions. Having MFS significantly decreased the odds of experiencing no difficulties on all respective dimensions $(\mathrm{P}<0.05)$, except for self-care. Similar results are reflected in Table 3, which compares the subscales of the EQ-5D-5L for the MFS sample and for the chosen comparison group of ACHD.

Compared to the larger ACHD group, patients with MFS reported significantly worse QOL in usual activities $(\mathrm{P}=0.002)$, pain/discomfort $(\mathrm{P} \leq 0.001)$, and anxiety/ depression $(\mathrm{P}=0.022)$. Observed differences were most extreme on the pain/discomfort dimension. No significant differences were observed on the self-care $(\mathrm{P}=0.483)$ or mobility $(\mathrm{P}=0.059)$ dimensions. Figure 1 is a graphic representation of the comparison between the 5 dimensions of the EQ-5D-5L for both samples.

When comparing the combined measures of QOL between patients with MFS and non-MFS ACHD (Table 4), patients with MFS scored markedly lower on the VAS $(\mathrm{P}=0.073)$ and also had lower descriptive index values $(\mathrm{P}=0.025)$. However, the observed differences between the populations were less extreme in regard to the VAS scores. Additionally, the observed variations in QOL depended on the type of measurement applied. Accordingly, the mean VAS score was markedly lower than the descriptive index value for QOL.

\section{Discussion}

In recent decades, substantial medical advancements have improved the lives of individuals with MFS. Consequently, many clinicians count MFS among the most manageable of genetic conditions today (7). However, many affected patients do not share this view and describe considerable impairments in their QOL (4). Remarkably few studies have been undertaken to assess psychosocial consequences of MFS. The major purpose of this study was to explore QOL among individuals with MFS and elaborate on previous findings by utilizing the EQ-5D-5L, a highly reliable and valid outcome measure within the cardiovascular area (18). It includes two types of measurement, a short descriptive system questionnaire and a VAS, and therefore holds a more global view on QOL in terms of general life satisfaction. The current study provides evidence that patients with MFS are at particularly high risk for a diminished QOL and 
Table 1 Characteristics of the study populations

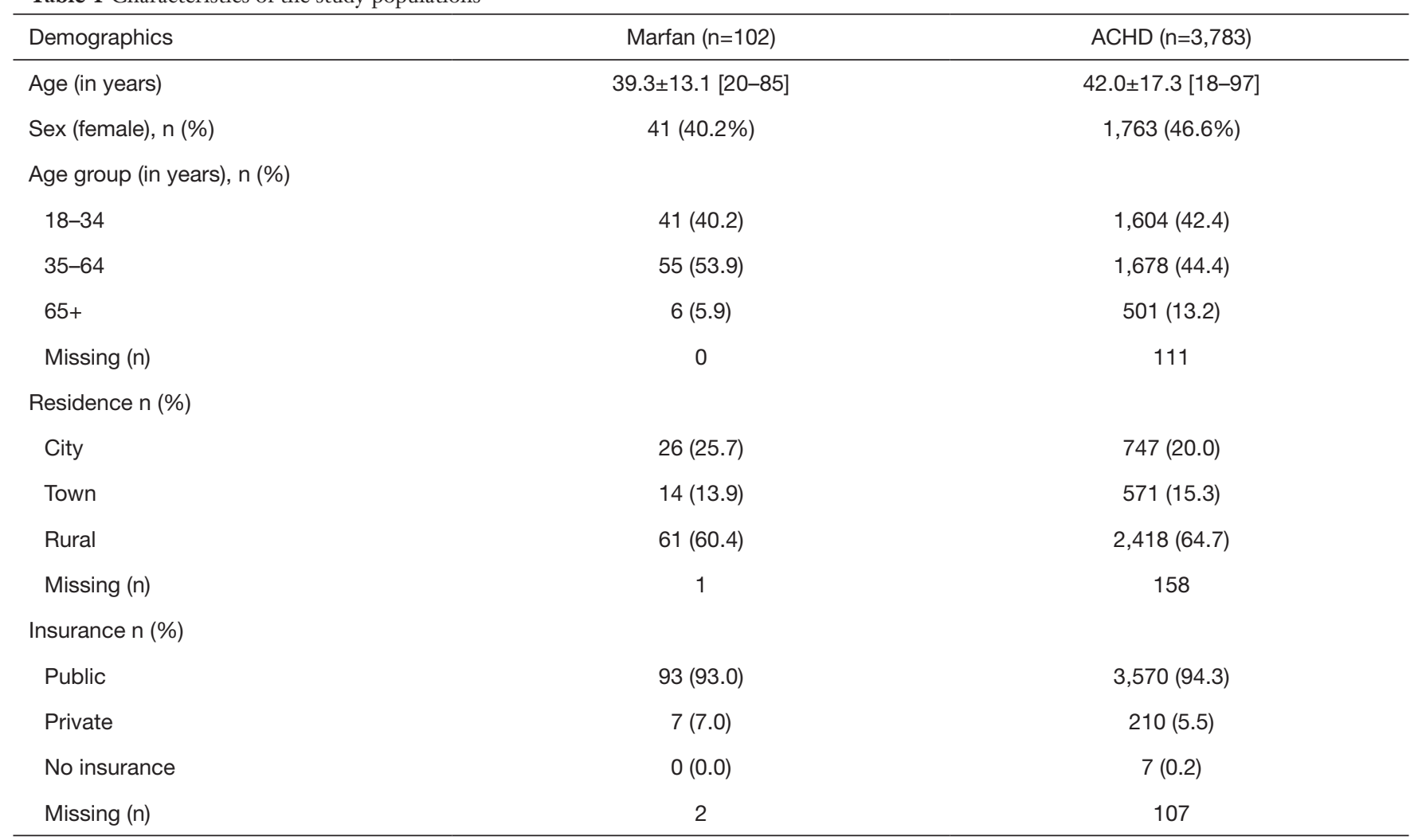

$\mathrm{ACHD}$, adults with congenital heart defects; $n$, absolute number.

Table 2 Impact of Marfan-diagnosis with respect to EQ-5Ddimensions

\begin{tabular}{lc}
\hline EQ-5D-dimensions & Value \\
\hline Mobility & $0.600^{*}(0.37-0.96)$ \\
Self-Care & $1.070(0.41-2.77)$ \\
Usual activities & $0.487^{*}(0.32-0.75)$ \\
Pain/discomfort & $0.370^{*}(0.25-0.55)$ \\
Anxiety/depression & $0.598^{*}(0.40-0.90)$
\end{tabular}

Displayed is the odds ratio, upper and lower bounds $(95 \% \mathrm{Cl})$ respectively which was obtained from several ordered logistic regressions using $\mathrm{EQ}$-dimensions as dependent variable. *, $\mathrm{P}<0.05$.

present major mental and physiological impairments.

The study encompassed a nationwide sample of patients with MFS recruited by the German Heart Center Munich. It was ensured that the reference data were ascertained with the same methods to ensure maximum comparability between the two populations.
Nonetheless, the present study may be subject to certain limitations. First, the study was cross-sectional in nature and does not allow any conclusions to be drawn regarding the etiology of psychological and physiological effects or the development in QOL over time. Second, as data were assessed retrospectively, it was not possible to determine precise response rates among the respondents. Third, the study relied on self-report outcomes and might be subject to recall and self-presentation bias. Fourth, as the enrolment was voluntary, selection bias could not be excluded and may limit representativeness. Fifth, this study was performed at tertiary care centers for ACHD which does not reflect the typical population of CHD patients, who most often present to non-specialized physicians. Sixth, the data derived only from patients living in Germany and generalization of the conclusions to the greater, global MFS population is debatable. Finally, since no control group was involved in the original study design, data could only be compared to the remaining respondents affected by a variety of types of CHD. In order to put findings into appropriate perspective, control group designs are conceivable in future clinical trials. 
Table 3 Distribution of EQ5D-dimensions



*, significant finding. ACHD, adults with congenital heart defects; $n$, absolute number. 


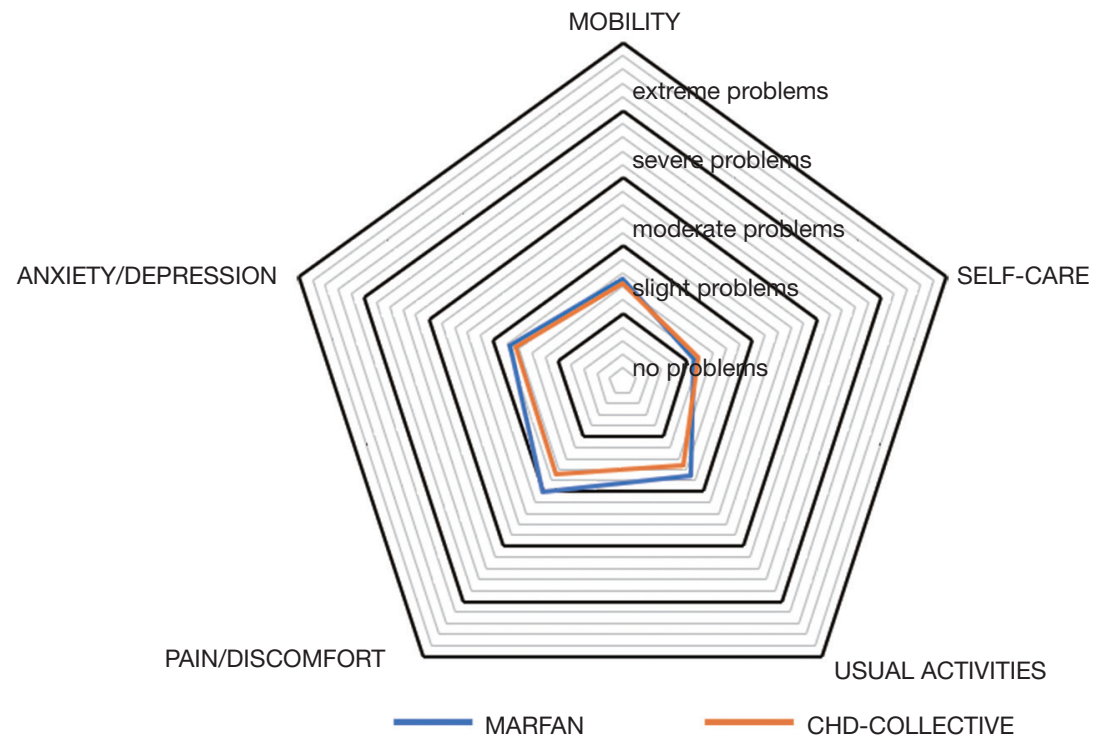

Figure 1 EQ-5D-5L values in the Marfan syndrome patients vs. the ACHD comparison group. Each dimension consists of 5 levels: no problems, slight problems, moderate problems, severe problems and extreme problems. Average values were calculated for all dimensions and accordingly depicted. ACHD, adults with congenital heart defects.

Table 4 Crosswalk Index Value vs. Visual Analog Scale (VAS)

\begin{tabular}{lccc}
\hline & Marfan (mean \pm SD) & ACHD (mean \pm SD) & P value \\
\hline Crosswalk & $86.97 \pm 15.12$ & $90.67 \pm 15.51$ & $0.025^{*}$ \\
VAS & $72.58 \pm 15.95$ & $76.23 \pm 19.04$ & 0.073 \\
\hline
\end{tabular}

*, significant finding. ACHD, adults with congenital heart defects; SD, standard deviation.

\section{QOL in patients with MFS}

\section{Pain/discomfort}

Current findings indicate that QOL of patients with MFS is significantly compromised in the physical domain compared to counterparts with other forms of CHD. Indeed, previous research has demonstrated that the percentage of MFS patients suffering from chronic pain ranges from $47 \%$ to $92 \%$ and that this is a significant and persistent problem in MFS (19). According to recent findings related to pain in individuals with MFS, pain is a major contributor to decreased QOL (4). The most severe types of pain include back pain followed by neck pain and headaches (20). Clinical manifestations of MFS, such as dural ectasia, degenerative disk disease, kyphosis and early osteo-arthritis frequently cause great pain (4). However, treatment options for chronic pain management are still lacking and research efforts in this area appear fragmented. In a survey of 993 patients with MFS, Nelsen et al. noted that very few patients receive medical procedures for pain and less than a half of them are satisfied with their current pain treatment (21). These findings are especially worrying since chronic pain is linked to profound disability and significant psychological burden (19). Further research is needed to characterize, in greater depth, the directionality of pain, mental well-being and QOL in patients with MFS. Better management could potentially improve an individual's satisfaction with life by facilitating work participation and everyday activities.

\section{Usual activities}

According to the present findings, having MFS increased the risk of reporting problems with usual activities (e.g., work, study, housework, family or leisure activities). Generally, emotional distress and physical inactivity have been found to be significantly correlated with work participation $(22,23)$. Studies confirm that the employment 
rate among patients with MFS is considerably below that of the general population $(24,25)$. Workplace discrimination and stigma are further factors that compound other difficulties in the daily lives of patients with MFS (26). Peters et al. found that $32 \%$ of adults with MFS reported feeling socially discriminated against and $20 \%$ of patients perceived instances of workplace discrimination. For this reason, these patients chose to withdraw from social situations or keep their illness secret $(7,26)$. Eighty percent of patients also reported reducing work hours due to MFS or missing on average of 6.5 to 7 months of work because of their treatment (20). Social stigmatization is significantly correlated with mental illness, pessimistic outlook towards MFS and low self-esteem (26). These findings indicate the necessity of offering individuals with MFS psychological assistance in order to facilitate their coping strategies and improve long-term outcomes.

\section{Mobility}

MFS was significantly associated with the occurrence of mobility impairments which is hardly surprising given the findings related to pain in patients with MFS. Symptoms commonly associated with MFS, such as chronic pain and fatigue, may lead to restricted mobility and lower QOL in the physical domain (27). Physical QOL issues have been found to be particularly related to age and the presence of scoliosis. Additionally, chronic pain was correlated with severe mental and physical fatigue which is ranked as one of the highest complaints in patients with MFS (20). Until now, it is unclear whether fatigue is linked to medication use, or inherent symptoms related to MFS itself (4). Further research is needed to investigate the exact etiology of fatigue in patients with MFS and eventually reveal modifiable features of MFS which could be considered in their treatment to improve their QOL.

\section{Anxiety/depression}

In accordance with earlier findings, patients with MFS experienced considerable impairments in the emotional area. Anxiety and depression have been recognized as biobehavioral variables, that have the strongest and most direct effects on QOL among all disease-related factors (28). Peters et al. found that over $40 \%$ of their study cohort had a significant degree of depressive symptoms (7). Emotional distress is known to have far-reaching implications for adverse psychological outcomes, such as body image dissatisfaction, negative illness perceptions and decreased self-esteem $(4,29)$, as well as negative cardiovascular consequences, including increased morbidity and premature mortality $(14,15)$. As patients sometimes present no observable physical signs, they frequently report feeling invisible in the health care system and struggle to find adequate treatment (7). Based on these findings, clinicians need to consider that psychological features that may lie beyond cardinal features of MFS may potentially play an equally important role in a patient's quality of life.

\section{Visual Analog Scale vs. Index values}

The two-fold measure of the EQ-5D-5L made it possible to investigate variations in QOL as determined by each of these two types of measurement. Apparently, the overall VAS score indicated a significantly lower QOL than that demonstrated by the descriptive index value, for both populations. It is conceivable that the descriptive system encourages a patient to examine QOL from different perspectives as it breaks down QOL into psychological, social, physical components. This goes along with Moons' operationalization of QOL as: "the degree of overall life satisfaction that is positively or negatively influenced by an individual's perception of certain aspects of life that are important to them, including matters both related and unrelated to bealth" (30). Thus, QOL is regarded as a multidimensional concept that is influenced by subjective evaluations of both positive and negative aspects of life (31). On the contrary, VAS picks up a one-dimensional view of perceived health, in which patients may be primarily focused on somatic health restrictions associated with their MFS.

\section{Conclusions}

Remarkably few studies have been undertaken to systematically assess psychosocial aspects of MFS. The present results provide evidence that individuals with verified MFS are likely to experience diminished QOL, in both mental and physical domains. Considerable restrictions in their QOL become even more evident when comparing the study group to patients with other forms of CHD. It is therefore important to consider the psychosocial consequences of MFS which cost resources for both, patients and professionals (e.g., time, money and emotional energy). Clinicians can help patients with MFS to reduce this cost by making mental health and psychosocial support an integral part of cardiac care for MFS. 


\section{Acknowledgments}

The authors thank the German Heart Foundation ("Deutsche Herzstiftung e.V."), the patient organization "Herzkind e.V.", and also the German health care insurance AOK-Bayern for the promotion of ACHD research.

Funding: This work was supported by the German Heart Foundation ("Deutsche Herzstiftung e.V.") (grant number F-30-15), the patient organization "Herzkind e.V." and the German healthcare insurance AOK-Bayern.

\section{Footnote}

Provenance and Peer Review: This article was commissioned by the editorial office, Cardiovascular Diagnosis and Therapy for the series "Current Management Aspects in Adult Congenital Heart Disease (ACHD): Part III". The article has undergone external peer review.

Reporting Checklist: The authors have completed the STROBE reporting checklist. Available at http://dx.doi. org/10.21037/cdt-20-692

Peer Review File: Available at http://dx.doi.org/10.21037/ cdt-20-692

Conflicts of Interest: All authors have completed the ICMJE uniform disclosure form (available at http:// dx.doi.org/10.21037/cdt-20-692). The series "Current Management Aspects in Adult Congenital Heart Disease (ACHD): Part III" was commissioned by the editorial office without any funding or sponsorship. HK serves as an unpaid editorial board member of Cardiovascular Diagnosis and Therapy from Feb 2018 to Jan 2022 and served as the unpaid Guest Editor of the series. The authors have no other conflicts of interest to declare.

Ethical Statement: The authors are accountable for all aspects of the work, including ensuring that questions related to the accuracy or integrity of any part of the work are appropriately investigated and resolved. The study was conducted in accordance with the Declaration of Helsinki (as revised in 2013). The survey has been approved by the institutional review boards of the Technical University Munich (157/16 S) and written informed consent was obtained from all participating patients before the start of documentation.
Open Access Statement: This is an Open Access article distributed in accordance with the Creative Commons Attribution-NonCommercial-NoDerivs 4.0 International License (CC BY-NC-ND 4.0), which permits the noncommercial replication and distribution of the article with the strict proviso that no changes or edits are made and the original work is properly cited (including links to both the formal publication through the relevant DOI and the license). See: https://creativecommons.org/licenses/by-nc-nd/4.0/.

\section{References}

1. von Kodolitsch Y, De Backer J, et al. Perspectives on the revised Ghent criteria for the diagnosis of Marfan syndrome. Appl Clin Genet 2015;8:137.

2. Cañadas V, Vilacosta I, Bruna I, et al. Marfan syndrome. Part 1: pathophysiology and diagnosis. Nat Rev Cardiol 2010;7:256.

3. Ratiu I, Virden TB, Baylow H, et al. Executive function and quality of life in individuals with Marfan syndrome. Qual Life Res 2018;27:2057-65.

4. Nielsen C, Ratiu I, Esfandiarei M, et al. A review of psychosocial factors of Marfan Syndrome: adolescents, adults, families, and providers. J Pediatr Genet 2019;8:109-22.

5. Kaemmerer H, Oechslin E, Seidel H, et al. Marfan syndrome: what internists and pediatric or adult cardiologists need to know. Expert Rev Cardiovasc Ther 2005;3:891-909.

6. Hansen LB, von Kodolitsch Y, Schroeder F, et al. Body Image in Patients with Marfan Syndrome. J Clin Med 2020;9:1015.

7. Peters KF, Kong F, Horne R, et al. Living with Marfan syndrome I. Perceptions of the condition. Clin Genet 2001;60:273-82.

8. von Kodolitsch Y, Demolder A, Girdauskas E, et al. Features of Marfan syndrome not listed in the Ghent nosology-the dark side of the disease. Expert Rev Cardiovasc Ther 2019;17:883-915.

9. Hunter AL, Swan L. Quality of life in adults living with congenital heart disease: beyond morbidity and mortality. J Thorac Dis 2016;8:E1632.

10. Handisides JC, Hollenbeck-Pringle D, Uzark K, et al. Health-related quality of life in children and young adults with Marfan syndrome. J Pediatr 2019;204:250-255.e1.

11. Rand-Hendriksen S, Johansen H, Semb SO, et al. Healthrelated quality of life in Marfan syndrome: a cross- 
sectional study of Short Form 36 in 84 adults with a verified diagnosis. Genet Med 2010;12:517-24.

12. Fusar-Poli P, Klersy C, Stramesi F, et al. Determinants of quality of life in Marfan syndrome. Psychosomatics 2008;49:243-8.

13. Gritti A, Pisano S, Catone G, et al. Psychiatric and neuropsychological issues in Marfan syndrome: a critical review of the literature. Int J Psychiatry Med 2015;50:347-60.

14. Watkins LL, Koch GG, Sherwood A, et al. Association of anxiety and depression with all-cause mortality in individuals with coronary heart disease. J Am Heart Assoc 2013;2:e000068.

15. Park JH, Tahk SJ, Bae SH. Depression and anxiety as predictors of recurrent cardiac events 12 months after percutaneous coronary interventions. J Cardiovasc Nurs 2015;30:351-9.

16. Herdman M, Gudex C, Lloyd A, et al. Development and preliminary testing of the new five-level version of EQ-5D (EQ-5D-5L). Qual Life Res 2011;20:1727-36.

17. Grochtdreis T, Dams J, König HH, et al. Health-related quality of life measured with the EQ-5D-5L: estimation of normative index values based on a representative German population sample and value set. Eur J Health Econ 2019;20:933-44.

18. Dyer MT, Goldsmith KA, Sharples LS, et al. A review of health utilities using the EQ-5D in studies of cardiovascular disease. Health Qual Life Outcomes 2010;8:13.

19. Speed TJ, Mathur VA, Hand M, et al. Characterization of pain, disability, and psychological burden in Marfan syndrome. Am J Med Genet A 2017;173:315-23.

20. Rao SS, Venuti KD, Dietz HC, et al. Quantifying health status and function in Marfan syndrome. J Surg Orthop Adv 2016;25:34-40.

21. Nelson AM, Walega DR, McCarthy RJ. The Incidence and Severity of Physical Pain Symptoms in Marfan Syndrome:
A Survey of 993 Patients. Clin J Pain 2015;31:1080-6.

22. Harder HG, Rash JA, Nelson S. Influences of labour participation among persons with disabilities: a systematic review and Best Evidence Synthesis. International Journal of Disability Management 2012;7:10-26.

23. Bertilsson M, Petersson EL, Östlund G, et al. Capacity to work while depressed and anxious-a phenomenological study. Disabil Rehabil 2013;35:1705-11.

24. Bathen T, Velvin G, Rand-Hendriksen S, et al. Fatigue in adults with Marfan syndrome, occurrence and associations to pain and other factors. Am J Med Genet A 2014;164A:1931-9.

25. Peters K, Kong F, Hanslo M, et al. Living with Marfan syndrome III. Quality of life and reproductive planning. Clin Genet 2002;62:110-20.

26. Peters KF, Apse K, Blackford A, et al. Living with Marfan syndrome: coping with stigma. Clin Genet 2005;68:6-14.

27. Schoormans D, Radonic T, De Witte P, et al. Mental quality of life is related to a cytokine genetic pathway. PLoS One 2012;7:e45126.

28. Moon JR, Cho YA, Huh J, et al. Structural equation modeling of the quality of life for patients with Marfan syndrome. Health Qual Life Outcomes 2016;14:83.

29. Helder MRK, Schaff HV, Lahr BD, et al. Quality of Life of Patients With Marfan Syndrome After Valve-Sparing or Valve-Replacement Operations. Mayo Clin Proc 2019;94:1906-9.

30. Moons P, Van Deyk K, Budts W, et al. Caliber of qualityof-life assessments in congenital heart disease: a plea for more conceptual and methodological rigor. Arch Pediatr Adolesc Med 2004;158:1062-9.

31. Moons P, Deyk KV, Bleser LD, et al. Quality of life and health status in adults with congenital heart disease: a direct comparison with healthy counterparts. Eur J Cardiovasc Prev Rehabil 2006;13:407-13.
Cite this article as: Andonian $\mathrm{C}$, Freilinger S, Achenbach S, Ewert P, Gundlach U, Kaemmerer H, Nagdyman N, Neidenbach RC, Pieper L, Schelling J, Weyand M, Beckmann J. Quality of life in patients with Marfan syndrome: a cross-sectional study of 102 adult patients. Cardiovasc Diagn Ther 2021;11(2):602-610. doi: 10.21037/cdt-20-692 